\title{
Preparation, Physico-Chemical Characterization and Pharmacodynamics of Ceftriaxone Loaded BSA Nanoparticles
}

\section{Bali GK*, Singla S, Kashyap Y, Dumka VK, Kalia A, Sharma M and Mehta N}

Department of Veterinary Pharmacology and Toxicology, Guru Angad Dev Veterinary and Animal Sciences University, Ludhiana, India

\begin{abstract}
The aim of the present study was to develop and characterize ceftriaxone loaded BSA nanoparticles. The nanoparticles were prepared by desolvation method. Scanning electron microscopy (SEM), transmission electron microscopy (TEM) and fourier transform infrared (FT-IR) characterization of the synthesized nanoparticles was done. SEM and TEM revealed that the nanoparticles had a smooth and spherical surface and FT-IR revealed that there was no interaction between the drug and the polymer. Encapsulation efficacy of nanoparticles was $44.8 \%$. The mean particle size of BSA obtained was $149.46 \pm 1.05 \mathrm{~nm}$, PDI was 0.09 and the zeta potential was $-28 \mathrm{mV}$. In vitro drug release at $\mathrm{pH} 7.4$ was found to be $85.8 \%$ at $12 \mathrm{~h}$ time period. Various mathematical models were used and the values nearest to R2 were evaluated. Model fitting revealed that it followed the Higuchi and Korsmeyer Peppas Model. The values of R were higher for Higuchi and Korsmeyers peppas model. Pharmacodynamic studies were done, for $S$. aureus the results of MIC and MBC of Drug were $2.51 \mu \mathrm{g}$ and $3 \mu \mathrm{g}$. The results of MIC and MBC of sample were $1.51 \mu \mathrm{g}$ and $2.1 \mu \mathrm{g}$ and for $E$. coli the results of MIC and MBC of Drug were $0.05 \mu \mathrm{g}$ and $0.08 \mu \mathrm{g}$. The results of MIC and MBC of sample were $0.05 \mu \mathrm{g}$ and $0.05 \mu \mathrm{g}$.
\end{abstract}

Keywords: Ceftriaxone; BSA nanoparticles; Encapsulation efficacy; Drug release

\section{Introduction}

Ceftriaxone is a third-generation cephalosporin that is used for a variety of infections such as meningitis, gonorrhoea and community-acquired pneumonia. The most important aspects of its pharmacokinetics include a long half-life, excellent tissue penetration and saturable (dose-dependent) serum protein binding of the drug. It is a time-dependent killer [1]. Incorporation of ceftriaxone nanoparticles has shown possible improvement in antimicrobial treatment, by enhanced intracellular delivery of the poorly cellpenetrating antibiotics. The nanoparticles are ingested by phagocytic system, involve macrophage activation and increase the immune response of the host [2]. In order to ensure the utility of nanoparticles it's important to have a thorough understanding of their physicchemical properties. The properties have shown to have a significant effect on particle stability, drug retention etc. The key advantages of nanoparticles are (1) improved bioavailability by enhancing aqueous solubility, (2) increasing resistance time in the body (increasing half life for clearance/increasing specificity for its cognate receptors and (3) targeting drug to specific location in the body (its site of action). This results in concomitant reduction in quantity of the drug required and dosage toxicity, enabling the safe delivery of toxic therapeutic drugs and protection of non target tissues and cells from severe side effects [3]. Nanoparticles are used as drug carriers in pharmacological industries with improved therapeutic effects and reduced toxic side effects [4].

\section{Materials and Methods}

\section{Drugs and chemicals}

Cetrioxone sodium salt (cefbact) marketed drug was obtained from Cipla limited, India. Bovine serum albumin (BSA) was obtained from Hi-Media Laboratories Pvt. Ltd. Mumbai. Acetone (analytical grade), was purchased from Merck (Mumbai, India).

\section{HPLC analysis}

Methanol $(\mathrm{MeOH})$, acetonitrile $(\mathrm{ACN})$ and orthophosphoric acid of HPLC grade were purchased from Merck (Mumbai, India).
Potassium dihydrogen orthophosphate was obtained from Loba Chemie Pvt. Ltd., Mumbai, India. Ultrapure analytical grade type 1 water for HPLC was produced by Milli-Q water purification system (Millipore, Bedford, MA, USA).

\section{Pharmacodynamic studies}

MacConkey Lactose Agar, Mueller Hinton Agar, Mueller Hinton Broth, Mannitol salt agar, Brain Heart Infusion Broth were all obtained from Hi-Media Laboratories Pvt. Ltd. Mumbai. Microwell plates (96 wells) with flat bottom of capacity $300 \mu$ were obtained from Orange Scientific, Belgium. The Tarsons India Pvt. Ltd. provided other materials like Eppendorf tubes, pipette tips and centrifuge tubes.

\section{Preparation of nanoparticles}

BSA nanoparticles: These were prepared by the desolvation method [5] with slight modification. BSA powder and drug (ceftrioxone) were added to distilled water and then acetone was added intermittently into $1 \%$ BSA solution at different $\mathrm{pH}(7,8,9)$ under stirring at room temperature until the solution became slightly turbid. $2 \mathrm{ml}$ of acetone was being added at every 5 minute interval, then few $\mathrm{ml}$ of $25 \%$ glutaraldehyde solution was added drop wise and the solution was then kept for 3 hours on continuous stirring for cross linking and the nanoemulsion was obtained.

\section{Characterization of nanoparticles}

Particle size: The particle size of nanoparticles was done by

*Corresponding author: Bali GK, Department of Veterinary Pharmacology and Toxicology, Guru Angad Dev Veterinary and Animal Sciences University, Ludhiana, India, Tel: 9463201126; E-mail: kaurg1050@gmail.com

Received: April 26, 2018; Accepted: July 13, 2018; Published: July 17, 2018

Citation: Bali GK, Singla S, Kashyap Y, Dumka VK, Kalia A, et al. (2018) Preparation, Physico-Chemical Characterization and Pharmacodynamics of Ceftriaxone Loaded BSA Nanoparticles. J Nanomed Nanotechnol 9: 509. doi: 10.4172/2157-7439.1000509

Copyright: (๑) 2018 Bali GK, et al. This is an open-access article distributed under the terms of the Creative Commons Attribution License, which permits unrestricted use, distribution, and reproduction in any medium, provided the original author and source are credited. 
dynamic light scattering. DLS measurements were performed using a dynamic light scattering instrument model Zetasizer Nano ZS (Malvern Instruments, Malvern, UK) having $632.8 \mathrm{~nm}$ red laser at $25^{\circ} \mathrm{C}$ at an angle of detection of $173^{\circ}$ with incubation time of 60 seconds in the round aperture glass cell.

Zeta potential: Preparation of the samples was done by diluting the nano-suspension 1:50 times and then estimating the size and zeta potential. The size analysis was done in a ZS 90 Malvern cuvette which was put in the analysis chamber to get various peaks and next to find its average zeta size. For zeta potential analysis a different cuvette was used. Accordingly the results of $\mathrm{z}$-average diameter, polydispersity index (relative width of the particle size distribution) and zeta potential of the nano-dispersion were recorded.

Particle shape: Nano suspensions particle shape is determined by electron microscopy either scanning electron microscopy (SEM) or transmission electron microscopy (TEM). In order to form the solid particles from nano suspensions these are mostly subjected to lyophilisation / freeze drying. These techniques play an important role in validating the reliability of other routine particle sizing techniques such as dynamic light scattering. SEM and TEM gives us the detailed nano and microscopic 2D images. TEM derives internal composition details such as crystallinity and lattice structure, SEM is suited to looking at samples surface characteristics. Image analysis gives us particle size data for individual nanoparticles, number based size distribution for the entire dispersion and various shape and morphological parameters.

Scanning electron microscopy (SEM): This electron microscopy based technique determines the size, shape and surface morphology with direct visualization of the nanoparticles. Therefore scanning electron microscopy offer several advantages in morphological and sizing analysis. However they provide limited information about the size distribution and true population average. During the process of SEM characterization, solution of nanoparticles should be initially converted into a dry powder. This dry powder is then further mounted on a sample holder followed by coating with a conductive metal (e.g. gold) using a sputter coater. Whole sample is then analyzed by scanning with a focused fine beam of electrons. Secondary electrons emitted from the sample surface determine the surface characteristics of the sample. This electron beam can often damage the polymer of the nanoparticles which must be able to withstand vacuum. Average mean size evaluated by SEM is comparable with results obtained by dynamic light scattering [1].

Transmission electron microscopy (TEM): For TEM the instrument was Hitachi $\mathrm{H}-7650$ with $40-120 \mathrm{kV}$ accelerating voltage; $0.2 \mathrm{~nm}$ resolution; magnification range $200 \mathrm{x}-200000 \mathrm{x}$ in High contrast (HC) mode; 4000x-600000x in high resolution mode (HR); tungsten and $\mathrm{LaB}_{6}$ Filaments; $1024 \times 1024$ pixels digital camera was used for imaging of the sample. The sample to be estimated in TEM was prepared by centrifuging $1 \mathrm{ml}$ of the suspension at high speed and then resuspending the pellet in deionized water. This was then put onto the support grids, allowed to dry and then viewed.

For SEM the instrument used was Hitachi S-3400 N with 0.3-30 $\mathrm{kV} ; 3 \mathrm{~nm}$ resolution at $30 \mathrm{kV}$ with secondary electron (SE) detector in high vacuum mode. Lyophilized powdered samples were used for the imaging. The samples of SEM were then sputtered onto the grid using ion sputter coater. Hitachi model E-1010 with gold and carbon coating units was used. The coating was done to prevent image distortion due to charging up of the specimen surface.

FTIR spectroscopy: For studying the nature of surface adsorbents in nanoparticles, they mostly have large surface area their modification by suitable adsorbate generates different properties. The property change between the nanoparticles containing adsorbates and bare nanopartcles can easily be detected by FTIR spectroscopy. All the experiments were performed using Thermo Nicolet 6700 Fourier Transform Infrared (FT-IR). The sample for IR was completely dried in hot air oven, were mixed with $\mathrm{KBr}$ powder and pelletized before performing the scan from wave number $4000 \mathrm{~cm}^{-1}$ to $400 \mathrm{~cm}^{-1}$.

Drug entrapment efficiency: The amount of the drug that is incorporated in the particles depends on the capacity of the lipid to dissolve/disperse it. Drug entrapment efficiency influences the release characteristics of drug molecule. The drug encapsulation amount per unit weight of nanoparticles is done by separating the entrapped drug from formulation. The separation is done by techniques such as ultracentrifugation, gel permeation chromatography and centrifugation filtration. The nanoparticles were ultracentrifuged at $15,000 \mathrm{rpm}$ for 1 hour 30 minutes and temperature was maintained at $4^{\circ} \mathrm{C}$ in order to separate them from aqueous medium. To remove the unentrapped drug molecules the supernatant was decanted and then it was dispersed into phosphate buffer saline $\mathrm{pH}$ 7.4. The procedure was repeated twice for complete removal of unentrapped drug molecules. The difference between the amount of drug used to prepare nanoparticles and amount of drug present in the aqueous medium gives the amount of entrapped drug into the nanoparticles.

Drug entrapment efficiency (\%): Amount of drug released from nanoparticles after centrifugation/Total amount of drug used to prepare nanoparticles.

Drug release kinetics: The most common methods for the determination of release kinetics from nanoparticle drug delivery systems is dialysis. For drug release from the nanoparticles, the nanoemulsion was incooperated into the dialysis bag which was regenerated first by washing under running water, soaked in PBS pH7.4 and was then incubated for 24 hours at $37^{\circ} \mathrm{C}$. The dialysis bag containing $1 \mathrm{ml}$ of nanoemulsion was then kept in $30 \mathrm{ml} \mathrm{PBS}$ at $\mathrm{pH}$ 7.4 from which the sample was collected at various time intervals 0 , $30 \mathrm{~min} 1 \mathrm{~h}, 2 \mathrm{~h}, 4 \mathrm{~h}, 6 \mathrm{~h}, 8 \mathrm{~h}, 10 \mathrm{~h}$ and $12 \mathrm{~h}$ and analysed in HPLC. The PBS in the sink receiver compartment was replaced entirely at every sampling interval. In dialysis, the appearance of drug in the "sink "receiver compartment containing PBS is the result of diffusion from the nanoparticles followed by diffusion across the dialysis membrane.

HPLC (High Performance Liquid Chromatography): The estimation of ceftrioxone in nanoparticle emulsion was done by using high performance liquid chromatography (HPLC) as per the method of Patel et al. [6] with slight modification.

Chromatographic conditions: The analytical column used was $\mathrm{C}_{18}$ column (Sun Fire, Particle size $5 \mu \mathrm{l}, 4.6 \times 150 \mathrm{~mm}$, Waters, USA). The mobile phase phosphate buffer $\left(\mathrm{P}^{\mathrm{H}} 3.5\right)$ : methanol 60:40 v/v was pumped through the column at a flow rate of $1 \mathrm{ml} / \mathrm{min}$. The sample injection volume was $100 \mu \mathrm{l}$. The detector was set to a wavelength of $254 \mathrm{~nm}$ and the chromatographic run time was set to 20 minutes.

Mobile phase was filtered, degassed by passage through a $0.45 \mu \mathrm{m}$ nylon filter (Millipore, Bedford, MA) under vacuum and sonicated for 30 mins. The Total Chrome software ${ }^{\circledR}$ (version 6.1) was used for instrument control and data analysis.

Stock solutions and standards: Ceftrioxone standard was prepared by weighing the drug and then dissolving it in water. The standard stock solution concentration was $10 \mathrm{mg} \mathrm{ml}^{-1}$. This initial stock solution of the drug was diluted quantitatively with water for the preparation 
Citation: Bali GK, Singla S, Kashyap Y, Dumka VK, Kalia A, et al. (2018) Preparation, Physico-Chemical Characterization and Pharmacodynamics of Ceftriaxone Loaded BSA Nanoparticles. J Nanomed Nanotechnol 9: 509. doi: 10.4172/2157-7439.1000509

of standard concentrations. The samples were prepared fresh at concentration range between $0.1 \mu \mathrm{g}$ to $50 \mu \mathrm{g}$. processing of the samples was done before HPLC analysis using the above mentioned method.

Sample preparation procedure: Sample taken for Entrapment efficacy was a 10 fold dilution of the supernatant obtained and then it was filtered through a syringe filter. This filtrate was then analyzed in the HPLC system. No such dilutions were done while analyzing the drug content of the sample obtained during in vitro drug release estimation. The sample was filtered and injected into the HPLC system.

Pharmacodynamic Parameters: The Pharmacodynamic parameters i.e. MIC, MBC of ceftrioxone loaded nanoparticles against E. coli and S. aureus were determined in Mueller Hinton Broth and Brain Heart Infusion Broth using broth microdilution method [7] according to CLSI (2008) guidelines.

\section{Results and Discussion}

Three formulations of ceftriaxone loaded BSA nanoparticles were prepared at different $\mathrm{pH}$ and at $\mathrm{pH} 9$ the nanoparticles with the best size were obtained (Table 1).

\begin{tabular}{|c|c|c|c|}
\hline S. No & pH & Mean \pm std dev & PDI \\
\hline 1 & 7 & $191.66 \pm 1.65$ & $0.17 \pm 0.02$ \\
\hline 2 & 8 & $155.23 \pm 3.72$ & $0.14 \pm 0.03$ \\
\hline 3 & 9 & $149.46 \pm 1.05$ & $0.09 \pm 0.01$ \\
\hline
\end{tabular}

Table 1: Particle size of BSA.

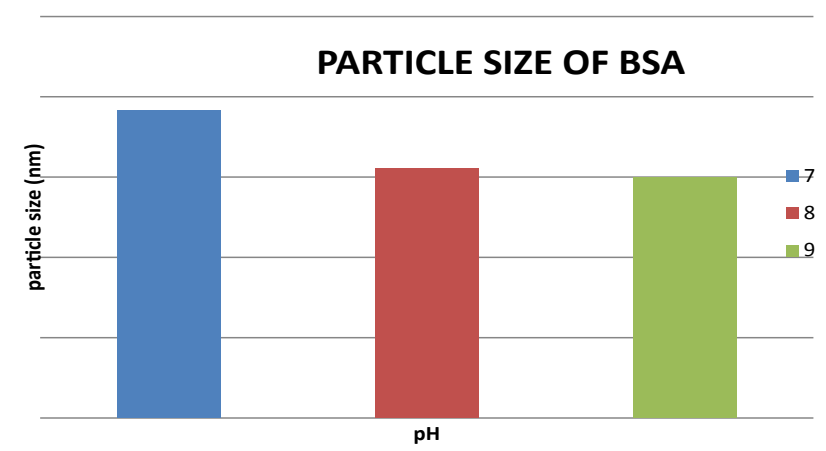

Figure 1: Comparison of mean particle size of three formulations.
The above data was analysed statistically using one way analysis of variance (post Hoc tukey's) depicting that there was no significant difference among the three formulations which indicates the efficiency of BSA to be used as a standard. It was observed at a particular $\mathrm{pH}$ intermittent addition of acetone improves reproducibility in the preparation of BSA nanoparticles. Acetone was added intermittently to $1 \%$ BSA solution at different $\mathrm{pH} 7,8,9$ and the best particle size was obtained at pH 9 (Figures 1-3). Similar finding was reported by Jun et al. [8].

\section{Physicochemical characterization of nanoparticles}

In our study, 149 to $191 \mathrm{~nm}$ size particles were obtained in all the formulations. Formulation having $\mathrm{pH} 9$ had a particle size of about $149.46 \pm 1.05 \mathrm{~nm}$ it was less compared to other formulations and the Zeta potential value was $-28 \mathrm{mV}$. SEM images showed the morphological properties of BSA nanoparticles. Well-defined spherical shaped particles were obtained. Under TEM also BSA nanoparticles were visualized as spherical nanoparticles, which are an agreement with the result, obtained using SEM (Figures 4 and 5) [9]

\section{FTIR (Fourier Transform Infrared)}

FTIR is an important study for the quick identification of encapsulated ceftriaxone molecules (Figures 5-7).

FTIR studies for Ceftriaxone showed characteristic peaks at $3432.7 \mathrm{~cm}^{-1}$ (N-H stretching mode of $\mathrm{H}$-bonded amide group), 1741 $\mathrm{cm}^{-1}$ ( $\beta$-lactam $\mathrm{C}=\mathrm{O}$ stretching vibrations) and $1592 \mathrm{~cm}^{-1}$ oxime $\mathrm{C}=\mathrm{N}$ stretching vibrations) [10].

For BSA the peaks were observed at $3381 \mathrm{~cm}^{-1}$ that assigned to the stretching vibration of $\mathrm{OH}$ (amide $\mathrm{A}$, mainly $\mathrm{NH}$ stretching vibration); at $2958 \mathrm{~cm}^{-1}$, at $2932 \mathrm{~cm}^{-1}$ and at $2873 \mathrm{~cm}^{-1}$ that approximate assigned $\mathrm{C}-\mathrm{H}$ and $\mathrm{C}-\mathrm{H}$ (methoxy compounds) stretching vibration; at 1642 $\mathrm{cm}^{-1}$ and at $1292 \mathrm{~cm}^{-1}$ that assigned to having approximate vibration of (NH) $\mathrm{C}=\mathrm{O}$ group and for $\mathrm{C}-\mathrm{C}$ and $\mathrm{C}-\mathrm{N}$ stretching respectively [11].

\section{Drug entrapment efficiency}

BSA nanoparticles can be potential alternative for drug delivery of ceftriaxone because it shows Entrapment efficacy of $44.8 \%$ which is quite reasonable to use for the delivery of this drug.

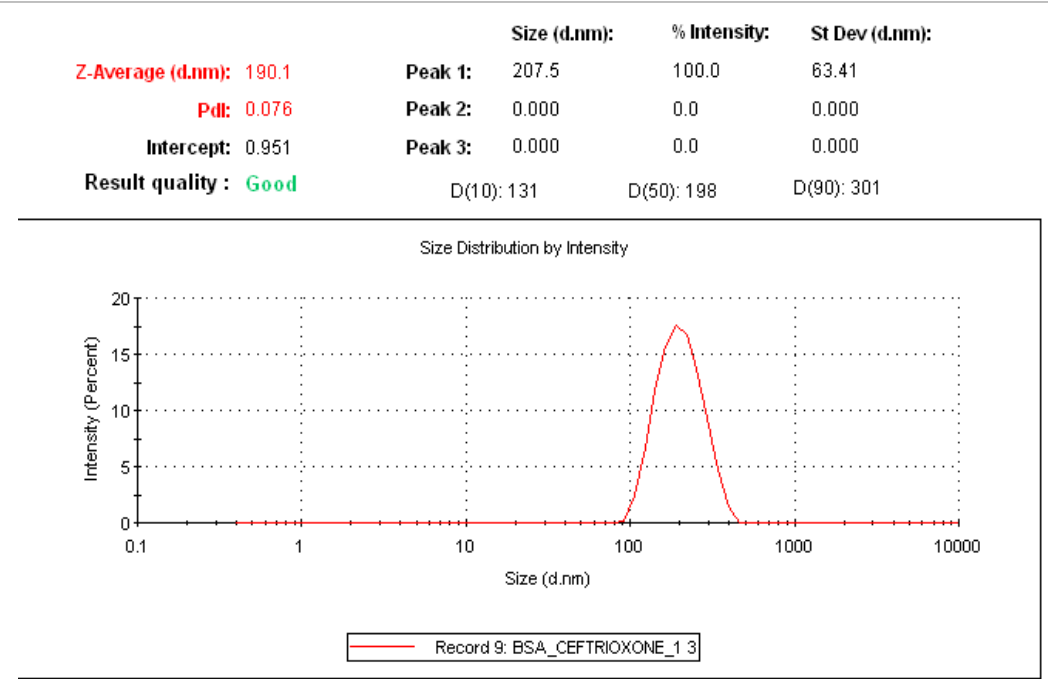

Figure 2: Particle size. 


\section{PDI OF BSA}

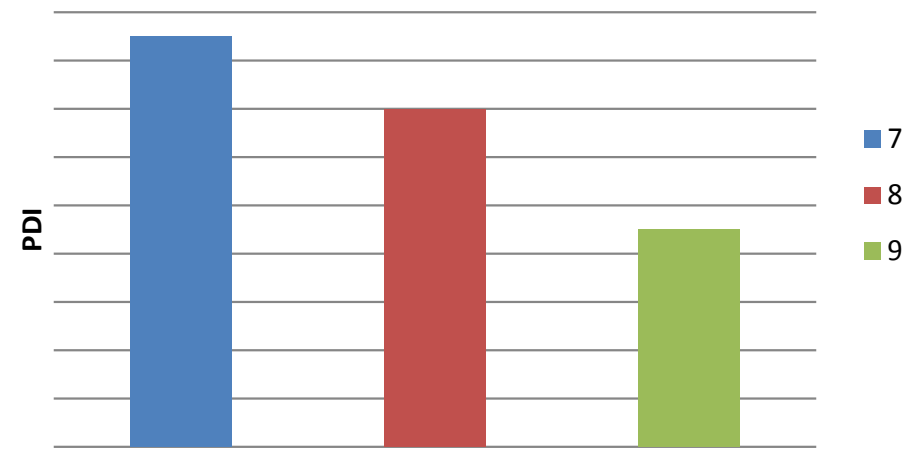

Figure 3: Comparison of mean PDI of three formulations.

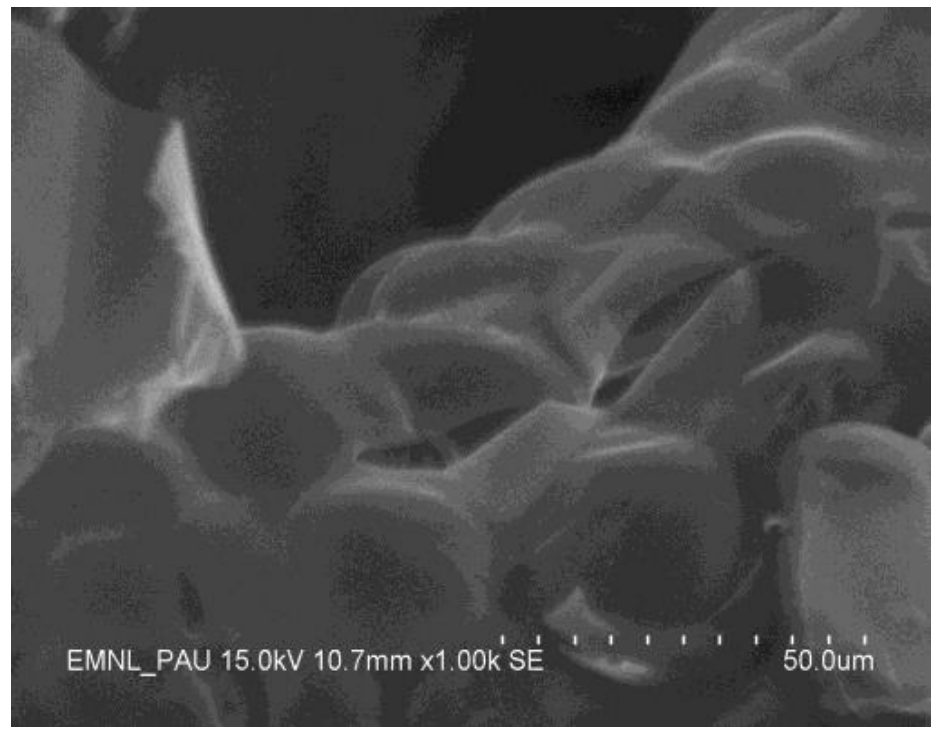

Figure 4: SEM image.

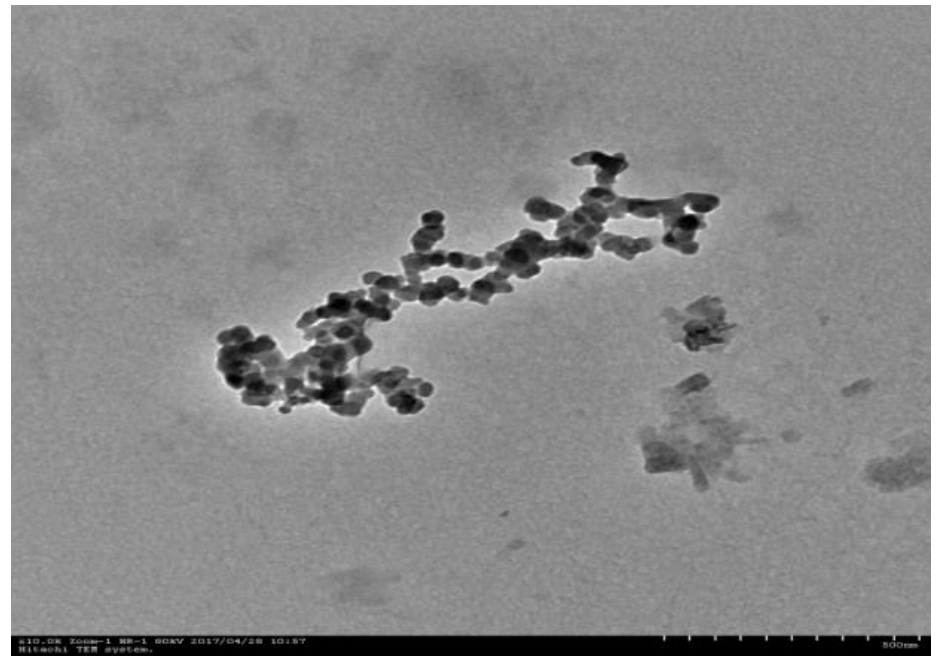

Figure 5: TEM image. 


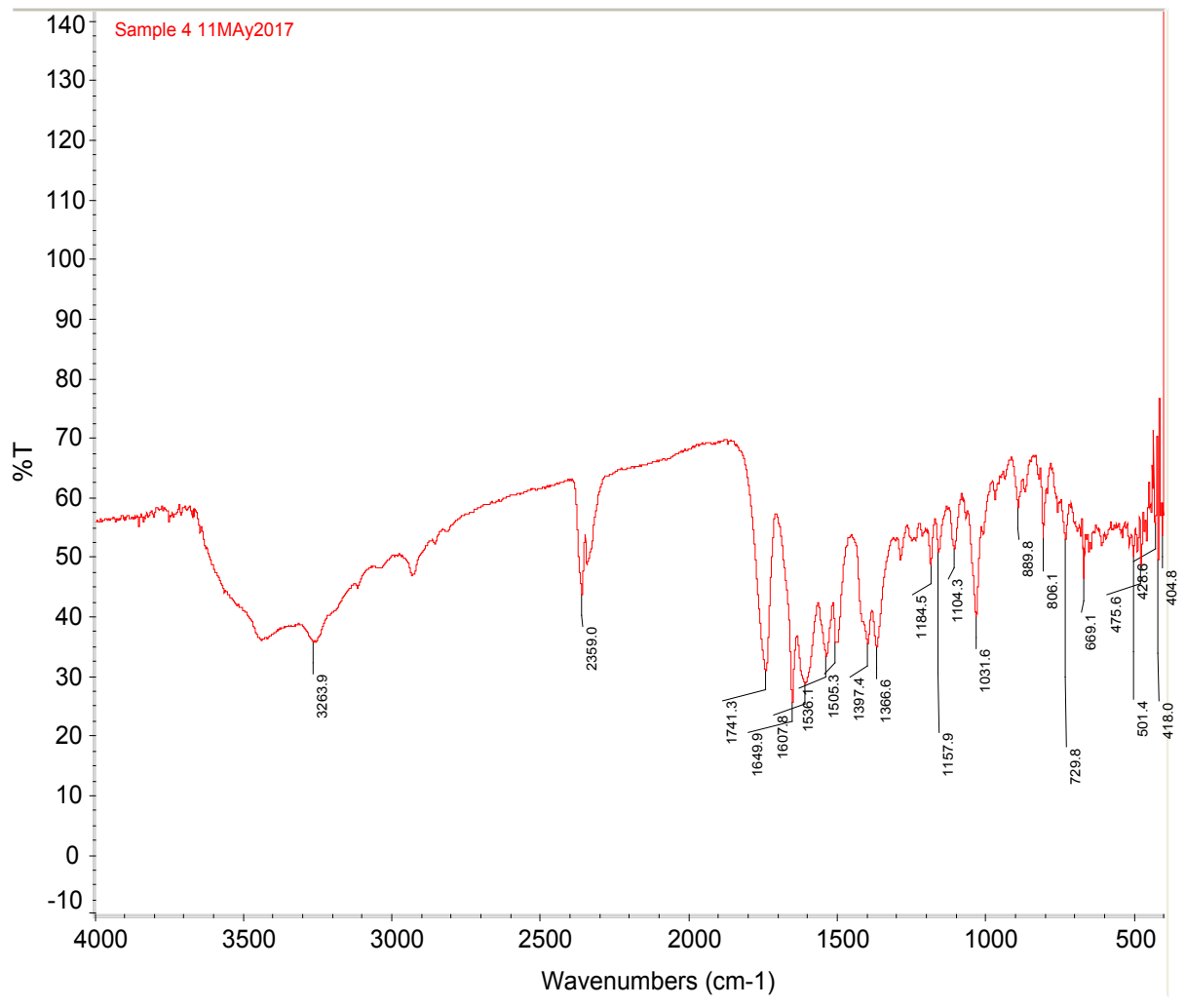

Figure 6: FTIR spectra of drug.

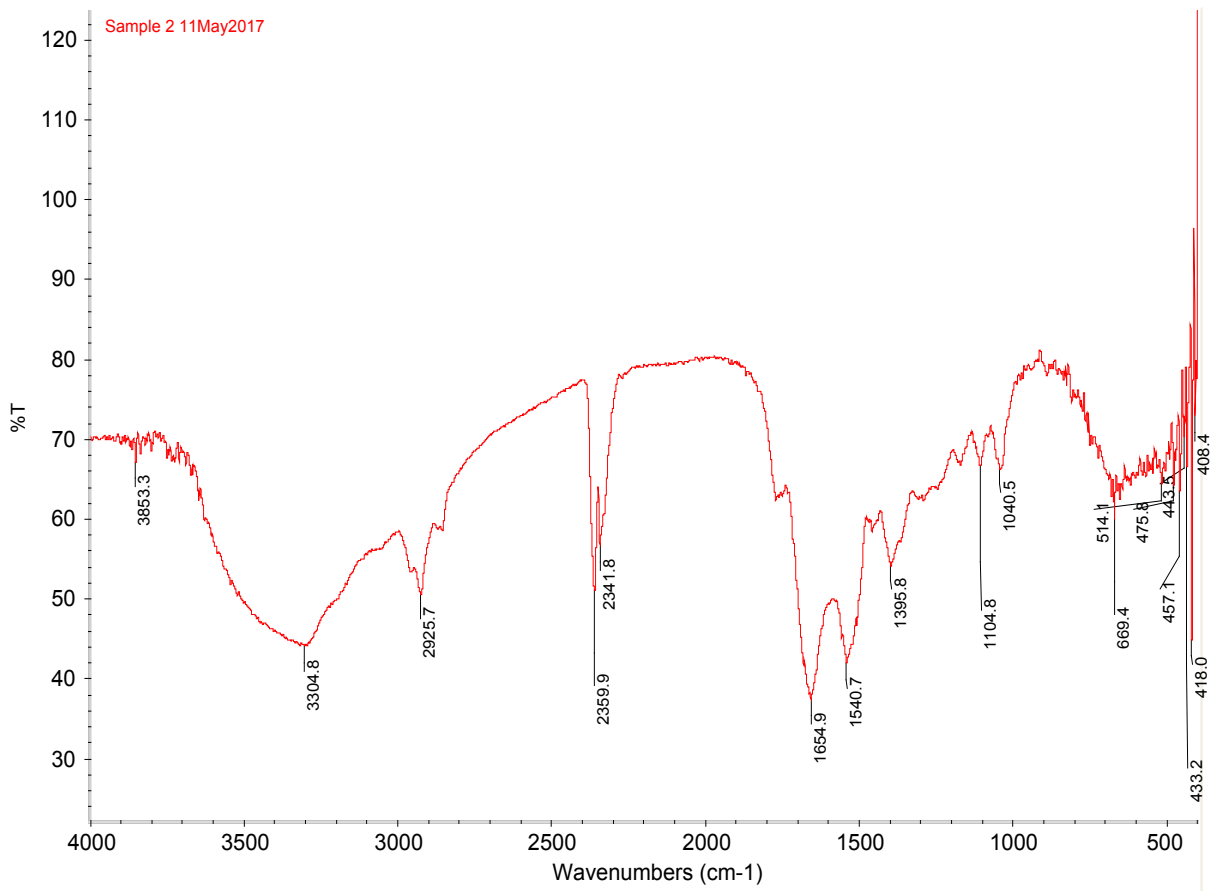

Figure 7: FTIR spectra of nanoparticles.

\section{In vitro release studies}

For drug release from the nanoparticles, the nanoemulsion was incooperated into the dialysis bag which was regenerated first by washing under running water, soaked in PBS pH7.4 and was then incubated for 24 hours at $37^{\circ} \mathrm{C}$. The dialysis bag containing $1 \mathrm{ml}$ of nanoemulsion was then kept in $30 \mathrm{ml} \mathrm{PBS}$ at $\mathrm{pH} 7.4$ from which the sample was collected at various time intervals $0,30 \min 1 \mathrm{~h}, 2 \mathrm{~h}, 4 \mathrm{~h}$, $6 \mathrm{~h}, 8 \mathrm{~h}, 10 \mathrm{~h}$ and $12 \mathrm{~h}$ and analysed in HPLC. The PBS in the sink 


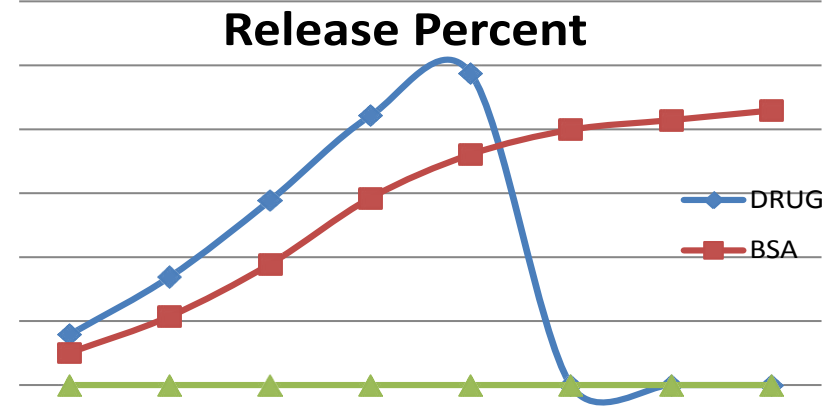

Figure 8: Data obtained from in vitro drug release studies were plotted as cumulative amount of drug released versus time.

receiver compartment was replaced entirely at every sampling interval. In dialysis, the appearance of drug in the "sink" receiver compartment containing PBS is the result of diffusion from the nanoparticles followed by diffusion across the dialysis membrane (Figure 8 ).

The data obtained from in vitro ceftriaxone release studies was treated by various mathematical models to determine the release mechanism. Selection of a suitable model was based on the values of $\mathrm{R}$ (Correlation Coefficient) obtained from the curves fitting of release data. Model fitting revealed that BSA followed the Higuchi and Korsmeyer Peppas Model. The values of $\mathrm{R}$ were higher for Higuchi and Korsmeyers peppas model [12]. The results in this study were consistent with the report described by Bansal et al. [13].

\section{Pharmacodynamic studies}

MIC and MBC determination for E. coli and S. aureus was done. Pharmacodynamic studies were done, for $S$. aureus the results of MIC and MBC of Drug were $2.51 \mu \mathrm{g}$ and $3 \mu \mathrm{g}$. The results of MIC and MBC of sample were $1.51 \mu \mathrm{g}$ and $2.1 \mu \mathrm{g}$ and for E. coli the results of MIC and $\mathrm{MBC}$ of Drug were $0.05 \mu \mathrm{g}$ and $0.08 \mu \mathrm{g}$. The results of MIC and MBC of sample were $0.05 \mu \mathrm{g}$ and $0.05 \mu \mathrm{g}$.

\section{Conclusion}

Nanoparticles were formed by Desolvation method and their further characterization and Pharmacodynamics was done. The average size of the nanoparticles was $149.46 \mathrm{~nm}$, PDI was 0.09 and the Zeta Potential was $-28 \mathrm{mV}$. SEM and TEM studies revealed they have a smooth and spherical surface. FTIR studies showed there was no interaction between the drug and polymer. Pharmacodynamic studies resulted in reduced value of MIC and MBC as compared to normal drug as a result dose rate will be reduced.

\section{References}

1. Perry TR, Schentaq JJ (2001) Clinical use of ceftriaxone: a pharmacokineticpharmacodynamic perspective on the impact of minimum inhibitory concentration and serum protein binding. Clin Pharmacokinet 40: 685-694.

2. Zaki NM, Hafez MM (2012) Enhanced Antibacterial Effect of Ceftriaxone Sodium-Loaded Chitosan Nanoparticles against Intracellular Salmonella typhimurium. AAPS Pharm Sci Tech 13: 411-421.

3. Irving B (2007) Nanoparticle drug delivery systems. Inno Pharm Biotechnol 24 58-62.

4. Feng S, Mu L, Win KY, Huang G (2004) Current Medicinal Chemistry. This J Supports Open Access 11: 413

5. Patel J, Bhavsar A, Patel B (2015) Development and validation of HPLCUV method for simultaneous estimation of ceftriaxone and sulbactam in pharmaceutical dosage form. Inter J Pharma Res \& Review.

6. Langer K, Balthasar S, Vogel V, Dinauer N, Von Briesen H, et al. (2003) Optimization of the preparation process for human serum albumin (HSA) nanoparticles. International Journal of pharmaceutics 257: 169-180.

7. Jun JY, Nguyen HH, Paik SYR, Chun HSC, Kang BC, et al. (2011) Preparation of size-controlled bovine serum albumin (BSA) nanoparticles by a modified desolvation method. Food Chem 127: 1892-1898.

8. Sidhu PK, Landoni MF, Aliabadi FS, Lees P (2010) P K-P D integration and modeling of marbofloxacin in sheep. Res Vet Sci 88: 134-41.

9. Manimekalai P, Manavalan R (2015) Selection of excipients for the formulation of ceftriaxone sodium loaded chitosan nanoparticle through drug-excipien compatibility testing. Inter J Pharm Tech Res 8: 5-10.

10. Rani K (2016) Fourier transforms infrared spectroscopy (FTIR) spectral analysis nanoparticles (BSA NPs) and egg albumin nanoparticles (EA NPs) of BSA. Res J Chemical Sci 6: 29-36.

11. Peppas BL, Blanchette JO (2004) Advanced Drug Delivery Review 56: 1649.

12. Bansal A, Kapoor DN, Kapil R, Chhabra N, Dhawan S (2011) Design and development of paclitaxel-loaded bovine serum albumin nanparticles for brain targeting. Acta Pharm 61: 141-156.

13. Kumari A, Yadava SK, Yogesh B, Singh PB, Yadav SC (2010) Development of biodegradable nanoparticles for delivery of quercetin. Colloids and Surfaces $B$ Biointerfaces 80: 184. 